\title{
Epifisiólisis de fémur proximal: resultados y calidad de vida
}

Dres. Juan Filippini* ${ }^{*}$ Ignacio Pagano*, María Elena Pérez* ${ }^{\star}$ Johan von Heideken†

\section{Resumen}

Introducción: el impacto sobre la calidad de vida de los pacientes con deslizamientos de epífisis femoral proximal inestables y estables es poco conocido. El objetivo de este estudio fue conocer los resultados terapéuticos utilizando un score de calidad de vida y las complicaciones de la población afectada en un centro de referencia ortopédico.

Material y método: estudio de cohorte que incluyó 28 niños tratados en el Centro Hospitalario Pereira Rossell entre 2010 y 2016. Se evaluaron los pacientes clínica y radiológicamente con un mínimo de dos años de seguimiento posoperatorio. Fue utilizado el cuestionario International Hip Outcome Tool (HOT-12), para medir resultados reportados por el paciente. Se evaluaron las complicaciones necrosis avascular, condrolisis y pinzamiento femoroacetabular.

Resultados: se identificaron 38 caderas tratadas por deslizamiento epifisario femoral proximal; 28 clasificadas estables (74\%) y 10 inestables (26\%). La fijación in situ fue el tratamiento quirúrgico más frecuentemente utilizado. Al final del seguimiento se evaluaron 27 pacientes y el iHOT-12 mostró una diferencia significativa entre deslizamientos estables y deslizamientos inestables 70 (rango 38-95) y 86 (57-100); $p=0,017$ ), respectivamente. No se observó necrosis avascular ni condrolisis y el pinzamiento femoroacetabular fue de $19 \%$ ( $n=7$ caderas; 6 estables y 1 inestable).

Conclusiones: los resultados clínicos de calidad de vida a través de scores reportados por los pacientes (PROM) utilizados eran significativamente mejores en los deslizamientos de la epífisis femoral proximal (DEFP) inestables en comparación con Ios DEFP estables. La ausencia de necrosis avascular en caderas inestables y la mayor proporción de pinzamiento femoroacetabular en los deslizamientos estables, a pesar de una remodelación femoral notoria luego de fijación in situ, podría explicarnos estos hallazgos inesperados.

Palabras clave: Epífisis desprendida de cabeza femoral

Niño

Calidad de vida

Key words: $\quad$ Slipped capital femoral epiphyses

Child

Quality of life

* Clínica de Traumatología y Ortopedia Pediátrica. Facultad de Medicina, Universidad de la República, Montevideo, Uruguay.

† Departamento de Salud del Niño y de la Mujer. Instituto Karolinska, Estocolmo, Suecia.

Estudio llevado a cabo en la Clínica de Traumatología y Ortopedia Pediátrica, Centro Hospitalario Pereira Rossell, Facultad de Medicina, Univer-

sidad de la República. Montevideo, Uruguay.

Los autores declaran no tener conflicto de interés

Correspondencia: Dr. Juan Filippini. Centro Hospitalario Pereira Rossell. Bulevar Gral. Artigas 1550, CP 11600 Montevideo, Uruguay.

Correo electrónico: jfilippini02@gmail.com

Aprobado por el Comité de Ética del CHPR.

Recibido: 21/1/20

Aprobado: 16/3/20 


\section{Introducción}

El deslizamiento de la epífisis femoral proximal (DEFP) es el trastorno de cadera más frecuente en adolescentes. Su incidencia varía entre 2-3/100.000 a 81/100.000 habitantes, dependiendo de las características de la población estudiada ${ }^{(1)}$. Por lo general, ocurre en la etapa de crecimiento rápido en niñas de 10-14 años y en niños de 12-16 años. La relación masculino-femenino es 2,4:1. Se cree que la etiología de DEFP es multifactorial, existiendo, además del sexo, edad y raza, factores mecánicos (trauma, obesidad, el aumento de la retroversión del cuello femoral, verticalización de la fisis) y endócrinos ${ }^{(1,2)}$. La afectación bilateral varía entre $21 \%$ y $63 \%{ }^{(3,4)}$. Un estudio reporta un compromiso bilateral primario en $7 \%$ y secuencial en $21 \%{ }^{(5)}$. El desplazamiento típico corresponde al desplazamiento epifisario posteromedial (varo), siendo el posterolateral (valgo) muy infrecuente ${ }^{(6)}$. La clasificación de Loder, basada en la estabilidad de la fisis, es la más utilizada en la actuali$\mathrm{dad}^{(7)}$. El DEFP estable representa aproximadamente el $75 \%-90 \%{ }^{(5,8,9)}$. Los DEFP inestables son menos frecuentes, pero tienen una tasa de complicaciones más elevada $^{(9-11)}$. La prevalencia de necrosis avascular (NAV) es variable. Los estudios analizados difieren según el tipo, la gravedad y el tratamiento quirúrgico realizado, por tanto, no se pueden comparar directamente entre sí. Según Herngren y colaboradores, en un reciente estudio observan 23\% de NAV en DEFP inestables, comparado con $3 \%$ en DEFP estables ${ }^{(11)}$. La condrolisis es infrecuente, alrededor de $1 \%-7 \%$, y se desconoce su etiología ${ }^{(11,12)}$. El pinzamiento femoroacetabular (PFA), vinculado a la deformidad residual tipo CAM, puede conducir a artrosis a mediano y largo plazo con la necesidad prematura de remplazo total de cadera $^{(13)}$. Los estudios que analizan los resultados clínicos de DEFP mediante scores reportados por los pacientes (PROM) y logran medir la calidad de vida en estos pacientes son escasos ${ }^{(11,14-18)}$. International Hip Outcome Tool (iHOT-12) ha sido utilizado por autores que destacan la precisión que brinda para la evaluación de las caderas en pacientes jóvenes y activos ${ }^{(19-21)}$. Por tanto, el objetivo de este trabajo fue conocer los resultados terapéuticos utilizando iHOT-12 y las complicaciones de la población afectada por DEFP, diagnosticados $\mathrm{y}$ tratados en un centro de referencia ortopédico.

Como hipótesis del trabajo, planteamos que los DEFP inestables presentan más complicaciones y peores resultados clínicos que los DEFP estables.

\section{Material y método}

En primer lugar se obtuvo la aprobación del Comité de Ética en investigación del Centro Hospitalario Pereira
Rossell (CHPR). En Uruguay, la amplia mayoría de los niños con DEFP son tratados en el centro de referencia en ortopedia pediátrica del CHPR. Desde la base de datos quirúrgica, se identificaron 31 pacientes en el período 2010-2016, tres fueron excluidos dado que no se encontraron las historias clínicas.

Todos los participantes o familiar de un paciente menor dieron su consentimiento para participar, realizando un control clínico y radiológico, con un mínimo de dos años de seguimiento desde la cirugía ortopédica. Basados en datos de la historia clínica y en las radiografías se confirmó el diagnóstico de DEFP y se clasificaron las caderas según Loder: si el paciente era capaz de deambular, el DEFP se consideraba estable; si el paciente era incapaz de deambular, incluso con muletas, el DEFP se consideraba inestable ${ }^{(7)}$.

\section{Demografía}

Los datos demográficos recopilados fueron la edad en la cirugía para el DEFP y otras cirugías de cadera, tipo de procedimiento quirúrgico, lateralidad, enfermedad concomitante (sí / no) (en caso afirmativo, síndrome de Down, trastorno hormonal, enfermedad sistémica), fijación profiláctica de la cadera normal (sí / no), índice de masa corporal (IMC) al momento de la cirugía y en el seguimiento. Un IMC igual o mayor que el percentil 85 en niños y adolescentes y un IMC igual o mayor a 25 en adultos, se clasificó como sobrepeso. El tiempo desde el inicio de los síntomas hasta la cirugía se estratificó en retraso del paciente (tiempo desde el inicio de los síntomas hasta la primera visita al médico), retraso del médico (tiempo desde la primera visita al médico hasta la cirugía).

\section{Examen clínico}

Todos los pacientes se sometieron a un examen físico detallado. Se midió el rango de movimiento de la cadera (ROM) bilateralmente usando un goniómetro. El signo de Trendelenburg se utilizó para evaluar el deterioro de la abducción de cadera y la discrepancia en la longitud del miembro ${ }^{(22)}$. Para determinar la presencia de PFA anterior, el investigador flexionó pasivamente la cadera a 90 grados con el paciente en decúbito supino, seguido de aducción forzada y rotación interna. Se registró una prueba positiva si estos movimientos producían dolor agudo en la ingle (TestPFA+) ${ }^{(23)}$. Se completó el instrumento internacional de resultados de la cadera (iHOT-12).

\section{Examen radiológico}

Todas las radiografías pre y posoperatorias fueron revisadas para confirmar la presencia de DEFP. Se trazó el ángulo de Southwick (epifisodiafisario) en ambas cade- 


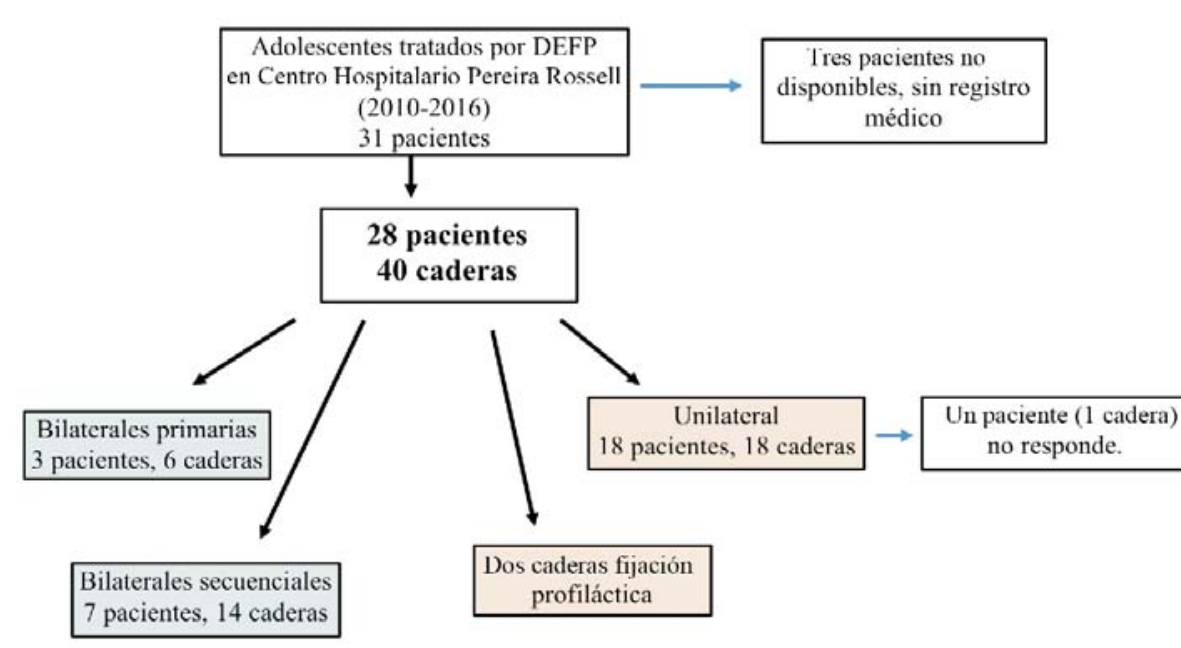

Seguimiento $>24$ meses

27 pacientes, 37 caderas

DEFP bilateral DEFP unilateral

10 pacientes 17 pacientes

Figura 1. Flujo de pacientes a través del estudio.

ras. La diferencia entre el lado afectado y el normal nos brindó la gravedad del deslizamiento, en caso bilateral se resta $12^{\circ}$ al ángulo medido. Fueron clasificados en: leve $\left(<30^{\circ}\right)$, moderada $\left(30^{\circ}\right.$ a $\left.60^{\circ}\right)$ y grave $\left(>60^{\circ}\right)^{(24)}$. Las mismas radiografías de la pelvis se obtuvieron en el seguimiento y se evaluaron para detectar el cierre fisario y las complicaciones. La precisión en la colocación del implante dentro de la epífisis se calificó de acuerdo con el método de Pring. Para una posición correcta, el implante tuvo que colocarse dentro del $50 \%$ central del ancho de la fisis, con la punta del tornillo a más de $5 \mathrm{~mm}$ a través de la fisis y en un ángulo de $70^{\circ}$ a $90^{\circ}$ con respecto a esta ${ }^{(25)}$. Además, en las radiografías posoperatoria inmediata y en la última radiografía se midió el ángulo alfa anterior ${ }^{(26)}$. Definimos síndrome de pinzamiento femoroacetabular cuando el paciente presentó dolor de cadera (TestPFA+) $\mathrm{y}$ ángulo alfa $>55^{\circ}$ (deformidad tipo CAM) ${ }^{(27)}$. Al espacio femoroacetabular menor de $3 \mathrm{~mm}$ o $\mathrm{ms}$ del $50 \%$ de reducción del espacio articular lo definimos condrólisis $^{(9)}$. Para definir NAV, utilizamos la clasificación radiológica de Ficat teniendo en cuenta la destrucción epifisaria, con estadios I a IV ${ }^{(28)}$.

\section{iHOT-12}

Este instrumento es recomendado para la evaluación de adultos jóvenes con dolor y disfunción de cadera ${ }^{(21)}$.
Consta de 12 preguntas en español, que son respondidas por el mismo paciente, con el objetivo de evaluar cuatro ámbitos: nivel de dolor y limitación funcional, deportes y recreación, impacto laboral y social, y, por último, emocional y estilo de vida ${ }^{(29)}$. Debido a la edad de los niños más pequeños en nuestro estudio, elegimos no usar una de las preguntas (¿Cuántos problemas tiene con la actividad sexual debido a su cadera?). La respuesta se realiza sobre una escala lineal, con un extremo expresando máxima función o mínimo dolor (100) y el extremo opuesto, mínima función y dolor extremo (0). El score total es calculado como un score promedio de las 11 preguntas (rango, 0 a 100, siendo 100 la mejor calidad de vida, con un mínimo de 14 puntos necesarios para evidenciar cambios clínicos) ${ }^{(20)}$.

Todas las evaluaciones clínicas y radiológicas fueron realizadas con un mínimo de 24 meses de seguimiento por uno solo de los autores (JF), el cual no estuvo involucrado en el tratamiento inicial y en el seguimiento previo de los pacientes.

\section{Análisis estadístico}

Se utilizaron estadísticas descriptivas, como conteos, porcentajes, medias con rangos para caracterizar los datos demográficos y la función subjetiva y objetiva de la cadera. Dos niños con DEFP bilateral secuencial habían 
Tabla 1. Características demográficas de niños con DEFP (número de pacientes $n=28$, número de caderas $n=38$ ).

\begin{tabular}{|c|c|}
\hline Variable & $n(\%)$ \\
\hline Edad promedio primera cirugía, (rango) & $12,1(9-14)$ \\
\hline Femenino & $13(48)$ \\
\hline $\begin{array}{l}\text { Tiempo desde el inicio de los síntomas hasta la } \\
\text { cirugía, semanas (rango) }\end{array}$ & $6(0-25)$ \\
\hline Retraso del paciente*, semanas (rango) & $3(0-25)$ \\
\hline Retraso del doctorł, semanas (rango) & $3(0-21)$ \\
\hline \multicolumn{2}{|l|}{ DEFP subtipo, número de caderas } \\
\hline Estable & $28(74)$ \\
\hline Inestable & $10(26)$ \\
\hline Pacientes con unilateral DEFP & $18(64)$ \\
\hline Derecha/izquierda & $8(44) / 10(56)$ \\
\hline Cirugía profiláctica en cadera contralateral & $2(12)$ \\
\hline Pacientes con bilateral DEFP & $10(26)$ \\
\hline DEFP bilateral primaria & $3(30)$ \\
\hline DEFP secuencial & $7(70)$ \\
\hline Enfermedades concomitantes & $20(77)$ \\
\hline Sobrepeso u obesidad $\ddagger$ & $25(89)$ \\
\hline Síndrome de Down & $1(4)$ \\
\hline Afectación hormonal & $1(4)$ \\
\hline Enfermedad sistémica & 0 \\
\hline \multicolumn{2}{|l|}{ Indicé masa corporal (kg/m²) } \\
\hline Días de cirugía, promedio (rango) & $26,0(18,00-36,8)$ \\
\hline Días de follow-up, promedio (rango) & $28,5(19,8-42,9)$ \\
\hline $\begin{array}{l}\text { * tiempo desde el inicio de los síntomas hasta } \\
\text { médico. } \\
\dagger \text { tiempo desde la primera visita al médico y } \\
+ \text { índice de masa corporal > percentil } 85 \text {. } \\
\text { § pacientes no disponibles para seguimiento. } \\
\text { DEFP: deslizamientos de la epífisis femoral }\end{array}$ & $\begin{array}{l}\text { primera visita al } \\
\text { ta la cirugía. } \\
\text { ximal. }\end{array}$ \\
\hline
\end{tabular}

presentado deslizamiento estable en una cadera y DEFP inestable contralateral. En estos pacientes, al calcular iHOT-12, se midió la primera cadera afectada. La prueba de Wilcoxon Signed Rank Test se utilizó para evaluar los cambios en los ángulos alfa anteriores. La prueba U de Mann-Whitney de muestras independientes se utilizó para las comparaciones entre iHOT-12 en el seguimiento para pacientes con DEFP estables e inesta- bles. Un valor de $\mathrm{p}<0,05$ se consideró estadísticamente significativo. El análisis estadístico se realizó con SPSS Mac 25.0 (SPSS Inc., Chicago, IL).

\section{Resultados}

De los 28 pacientes y 40 caderas incluidas en el estudio, no fue posible localizar a un paciente $(4 \%)$ para el seguimiento y dos presentaron fijación profiláctica. Por tanto, se evaluaron 27 pacientes y 37 caderas con un seguimiento promedio de 45 meses (24-87) (fgura 1). Los datos demográficos de 28 pacientes con DEFP tratados se obtuvieron de los registros médicos correspondientes; 15 varones y 13 mujeres; el promedio de edad al momento del diagnóstico fue de 12,1 años. Se presentaron como DEFP en varo el $100 \%$ de las caderas, clasificadas estables $74 \%(n=27)$ e inestables $26 \%(n=10)$. En el $89 \%$ de los pacientes se observó sobrepeso u obesidad al momento de la cirugía. El retraso diagnóstico fue prolongado, sea por retraso del paciente o del médico (tabla 1). Los pacientes con DEFP inestables recibieron tratamiento quirúrgico en menos de 24 horas después de la primera consulta de urgencia en nuestro hospital.

Se observaron 32 DEFP leves y moderados y 6 DEFP severos. Los pacientes con caderas inestables mostraban deslizamientos más severos que las estables.

Los procedimientos quirúrgicos fueron variados. Se realizó fijación in situ en mesa ortopédica (no reducción o reducción incidental) con un tornillo de rosca parcial en el $70 \%(n=7)$ de los DEFP inestables, reducción cerrada intencional en una cadera $(10 \%)$ y reducción abierta percutánea utilizando la técnica de Parsch en dos caderas inestables (20\%). Con el objetivo de disminuir la presión intracapsular se realizó la artroscentesis de cadera en 30\% ( $n=3)$ de los DEFP inestables. El protocolo de rehabilitación posoperatorio utilizado consistió en apoyo precoz progresivo para caderas estables y en las caderas inestables se retrasó la carga de peso durante seis semanas.

La posición del implante era correcta en $82 \%$ de las caderas estables y en $70 \%$ de las inestables.

Necesitaron reintervenciones cinco pacientes: tres para realizar retiro de osteosíntesis, de los cuales dos se debían al segundo tiempo de la técnica de Parsch y el restante por intolerancia al implante. Además, un paciente requirió limpieza quirúrgica por infección superficial del sitio quirúrgico y otro paciente presentó redesplazamiento, necesitando fijación suplementaria (tabla 2). La evaluación clínica y radiográfica ( $\mathrm{n}=27$ pacientes) se realizó con un seguimiento mínimo de 24 meses en el total de pacientes incluidos (tabla 3 ). Se destaca la diferencia significativa en los resultados de iHOT-12 total entre caderas con DEFP estable 70, rango (38-95) y DEFP inestable 86, (57-100), $\mathrm{p}=0,017$. 
Tabla 2. Características radiográficas y quirúrgicas de niños con DEFP (número de pacientes $\mathrm{n}=28$, número de caderas $n=38)$.

\begin{tabular}{|c|c|c|}
\hline Variable & $\begin{array}{l}\text { Caderas estables } \\
\qquad(n=28) n(\%)\end{array}$ & $\begin{array}{l}\text { Caderas inestables } \\
\quad(n=10) n(\%)\end{array}$ \\
\hline $\begin{array}{l}\text { Promedio ángulo de } \\
\text { desplazamiento enfoque } \\
\text { Lowenstein, (rango) }\end{array}$ & $26(7-60)$ & $50(30-70)$ \\
\hline Leve $\left(<30^{\circ}\right)$ & $17(61)$ & $1(10)$ \\
\hline Moderada $\left(30^{\circ}-50^{\circ}\right)$ & $9(32)$ & $5(50)$ \\
\hline Severa $\left(>50^{\circ}\right)$ & $2(7)$ & $4(40)$ \\
\hline $\begin{array}{l}\text { Artrocentesis } \\
\text { Técnica quirúrgica }\end{array}$ & 0 & $3(30)$ \\
\hline $\begin{array}{l}\text { Sin reducción o reducción } \\
\text { incidental (FIS) }\end{array}$ & $28(100)$ & $7(70)$ \\
\hline $\begin{array}{l}\text { Maniobra de reducción } \\
\text { intencional }\end{array}$ & 0 & $3(30)$ \\
\hline Reducción abierta & 0 & $2(67)$ \\
\hline Tipo de osteosíntesis & & \\
\hline Tornillo de rosca parcial & $27(96)$ & $7(70)$ \\
\hline Uno o más K-wire & 0 & $2(20)$ \\
\hline Tornillos con rosca total & $1(4)$ & $1(10)$ \\
\hline $\begin{array}{l}\text { Correcta posición de } \\
\text { osteosíntesis }\end{array}$ & $23(82)$ & $7(70)$ \\
\hline Reintervenciones & $3(11)$ & $2(20)$ \\
\hline Retiro de osteosíntesis & $1(4)$ & $2(20)$ \\
\hline $\begin{array}{l}\text { Revisión por infección de } \\
\text { herida }\end{array}$ & $1(4)$ & 0 \\
\hline Falla de implante & 0 & 0 \\
\hline $\begin{array}{l}\text { Desplazamiento } \\
\text { continuado }\end{array}$ & $1(4)$ & 0 \\
\hline
\end{tabular}

DEFP: deslizamientos de la epífisis femoral proximal.

Dada la deformidad provocada en esta patología, los pacientes pueden verse afectados en los movimientos de abducción (varo), flexión (CAM) y rotación interna (rotación externa). En esta serie la movilidad promedio para la flexión y rotación interna de las caderas controladas estuvieron dentro del rango de normalidad. Al momento del seguimiento el $22 \%(n=6)$ de DEFP estables con TPFA + tenían ángulo alfa $>55^{\circ}$ y $10 \%(\mathrm{n}=1)$ de DEFP inestables con TPFA + tenían ángulo alfa $>55^{\circ}$, por tanto, padecían PFA. Además, los pacientes con PFA ( $\mathrm{n}=7)$
Tabla 3. Examen clínico y radiográfico en seguimiento (número de pacientes $\mathrm{n}=27$, número de caderas $n=37)$.

\begin{tabular}{|c|c|c|}
\hline Variable & $\begin{array}{c}\text { Caderas } \\
\text { estables }(n=27)\end{array}$ & $\begin{array}{c}\text { Caderas } \\
\text { inestables }(n=10)\end{array}$ \\
\hline $\begin{array}{l}\text { Promedio tiempo de cirugía } \\
\text { hasta último examen clínico, } \\
\text { meses (rango) }\end{array}$ & $45(24-87)$ & $47(31-47)$ \\
\hline $\begin{array}{l}\text { Promedio tiempo desde } \\
\text { cirugía hasta última } \\
\text { radiografía, meses (rango) }\end{array}$ & $56(24-112)$ & $58(25-58)$ \\
\hline $\begin{array}{l}\text { Control radiográfico hasta } \\
\text { final de crecimiento, n (\%) }\end{array}$ & $27(100)$ & $9(90)$ \\
\hline \multicolumn{3}{|l|}{ Seguimiento clínico } \\
\hline $\begin{array}{l}\text { Promedio cadera afectada } \\
\text { máx. flexión, }{ }^{\circ} \text { (rango) }\end{array}$ & $121^{\circ}\left(100-140^{\circ}\right)$ & $123^{\circ}\left(100-140^{\circ}\right)$ \\
\hline $\begin{array}{l}\text { Promedio cadera afectada } \\
\text { máx. rotación interna, } \\
{ }^{\circ} \text { (rango) }\end{array}$ & $28^{\circ}\left(20-35^{\circ}\right)$ & $28^{\circ}\left(20-35^{\circ}\right)$ \\
\hline $\begin{array}{l}\text { Signo de Trendelenburg, } \\
\mathrm{n}(\%)\end{array}$ & 0 & 0 \\
\hline TPFA* positivo, n (\%) & $11(41)$ & $3(30)$ \\
\hline $\begin{array}{l}\text { Promedio ángulo alfa en Rx } \\
\text { posoperatoria inmediata } \\
\text { (cadera afectada), }{ }^{\circ} \text { (rango) }\end{array}$ & $60(50-90)$ & $61(50-70)$ \\
\hline $\begin{array}{l}\text { Promedio ángulo alfa en } \\
\text { última Rx (cadera afectada), } \\
{ }^{\circ} \text { (rango) }\end{array}$ & $53(45-65)$ & $56(45-65)$ \\
\hline $\begin{array}{l}\text { Ángulo alfa }>55^{\circ} \text { en la } \\
\text { cadera afectada en última rx } \\
+, n(\%)\end{array}$ & $9(33)$ & $2(20)$ \\
\hline $\begin{array}{l}\text { Ángulo alfa }>55^{\circ} \text { en caderas } \\
\text { con positivo TPFA, } n(\%)\end{array}$ & $6(22)$ & $1(10)$ \\
\hline Condrolisis última Rx , n (\%) & 0 & 0 \\
\hline NAV última Rx ${ }^{\dagger}, \mathrm{n}(\%)$ & 0 & 0 \\
\hline $\begin{array}{l}\text { NAV: necrosis avascular. } \\
* \text { TPFA=test de pinzamiento } \\
\dagger \text { Mínimo dos años de seguir }\end{array}$ & moroacetabula & \\
\hline
\end{tabular}

presentaron peor iHOT-12 62 (50-82) vs $81(38-100)$ en los 20 pacientes que no presentaron PFA $(\mathrm{p}=0,08)$. El seguimiento radiográfico demostró una remodelación significativa en el ángulo alfa, mejorando un promedio de $6^{\circ}(-5-40)$ en la cadera con DEFP $(\mathrm{p}=<0,001)$ en comparación con $3^{\circ}(-10-10)$ en la cadera normal $(\mathrm{p}=0,026)$. Las últimas radiografías mostraron que no hubo casos con complicaciones severas, principalmente no se detectaron signos de NAV (tabla 3 ). 


\section{Discusión}

Hasta donde sabemos, no hay estudios previos que comparen la calidad de vida de los pacientes con DEFP inestables y estables. Como era esperable, los deslizamientos estables fueron más frecuentes. En cambio, en comparación con otros estudios, se observó una alta proporción de DEFP inestables en nuestro trabajo ${ }^{(5,10)}$. Un resultado sorprendente fue que en promedio los DEFP estables estaban menos desplazados, pero los pacientes con DEFP inestables tuvieron mejores resultados de calidad de vida mediante iHOT-12. Además, los DEFP estables desarrollaron mayor proporción de PFA, lo que contrasta con lo informado tanto por Ulici y colaboradores, como por Lang y colaboradores ${ }^{(9)}$. A pesar de esto, los pacientes con PFA tuvieron un iHOT-12 promedio de 62, muy superior al observado en el estudio de Sansone y colaboradores, donde el iHOT-12 preoperatorio a una artroscopia de cadera por PFA era en promedio de $43^{(19)}$. El tratamiento llevado a cabo en DEFP inestables mediante reducción (incidental o intencional) del desplazamiento lograría restablecer la anatomía del fémur proximal, disminuyendo el ángulo de Southwick. En cambio, en los DEFP estables tratados con fijación in situ, el 100\% de los casos mantuvieron la deformidad. Esta deformidad residual podría conducir a un PFA y artrosis de la cadera a mediano y largo plazo ${ }^{(1,13,15,17)}$. A pesar de todo, la fijación in situ es el tratamiento más aceptado para DEFP en cualquier grado de severidad ${ }^{(5,8)}$. Si se preserva el crecimiento longitudinal del cuello femoral, muchos pacientes serán remodelados, disminuyendo los síntomas relacionados con $\mathrm{PF}^{(30-33)}$. El período de mayor remodelación se observa hasta el año posterior a la fijación ${ }^{(33)}$. Ortegren y colaboradores estudiaron fijación in situ con implantes diseñados para la fijación en las epífisis con una superficie lisa que atraviesa la fisis (Hansson hook-pin), demostrando mayor potencial de remodelación ${ }^{(33)}$. Según De Poorter y colaboradores, los DEFP leves y moderados tratados con fijación in situ están mostrando buenos resultados a largo plazo ${ }^{(16)}$. No obstante, Larson y colaboradores, tras un seguimiento a largo plazo (media 16 años), sostienen que uno de cada tres pacientes presentó dolor y el $12 \%$ requirió nueva cirugía de cadera ${ }^{(15)}$. Nectoux y colaboradores y Murgier y colaboradores declararon recientemente que un ángulo de deslizamiento inicial de aproximadamente 30-35 grados (moderados y severos) es el umbral en el cual el PFA aparecería más regularmente ${ }^{(17,34)}$. La alta tasa de PFA entre nuestros pacientes indicaría la necesidad de un posible tratamiento adicional, ya sea quirúrgico o no quirúrgico. Varias investigaciones demuestran que la NAV no es completamente conocida y probablemente multifactorial ${ }^{(12,35)}$. Algunos de los factores que podrían explicar el 0\% de NAV ob- servado en nuestra serie son: escasa proporción de DEFP severos, tratamiento quirúrgico precoz (menor de 24 horas desde la consulta en caderas inestables), descompresión articular mediante artroscentesis, cuidadosa técnica de fijación in situ utilizada en DEFP inestables y la adecuada colocación del tornillo transepifisario $^{(36)}$. Un aspecto interesante que se repite en las series es el retraso diagnóstico de DEFP, el cual podría influir en la severidad del desplazamiento y probablemente aumentaría el riesgo de inestabilización de la fisis, lo que puede llevar a mayor riesgo de complicaciones severas ${ }^{(37)}$. En tanto, el tratamiento profiláctico del DEFP continúa siendo controvertido. El posible acortamiento del cuello femoral y la NAV iatrogénica son dos argumentos en contra de la cirugía profiláctica de la cadera contralateral $^{(3,4)}$. En nuestro estudio, el $25 \%$ de los niños desarrollaron un deslizamiento secuencial en la cadera contralateral. Fue realizada fijación profiláctica en $12 \%$ de DEFP unilaterales. Si comparamos con Herngren $\mathrm{y}$ colaboradores, quienes informan hasta un $43 \%{ }^{(5)}$, parece existir un bajo porcentaje de tratamiento profiláctico en nuestro centro de referencia. Entonces, los niños que no se han sometido a cirugía profiláctica considerando el riesgo de deslizamiento secuencial en combinación con la demora del paciente y del médico, deberían programar un seguimiento radiográfico regular hasta el cierre de la fisis ${ }^{(3)}$. La indicación de fijación profiláctica actual se recomienda en pacientes con alto riesgo de deslizamientos subsiguientes. Algunos de los factores de riesgo son: edad $<10$ niñas $\mathrm{y}<12$ niños, obesos, con trastornos endócrinos, cadera asintomática con ángulo de Southwick entre $12^{\circ}$ y $15^{\circ}$ en el enfoque lateral y aquellos casos que tengan una baja probabilidad de seguimiento ${ }^{(12)}$. De forma similar a múltiples series publicadas, aquí se observó una alta prevalencia de sobrepeso u obesidad (89\%). Además, los pacientes mantienen el sobrepeso incluso entrada su adultez, demostrando la probable alteración metabólica de base ${ }^{(18)}$. Murray y Wilson confirmaron una estrecha correlación entre el aumento de la obesidad infantil en los últimos 20 años en Escocia y una incidencia creciente de $\operatorname{DEFP}^{(38)}$. Por otro lado, según Halvarson y colaboradores, la obesidad no es un factor suficiente para el desarrollo de DEFP, por lo que podría existir un factor independiente, como podría ser la hiperleptinemia, que sea capaz de explicar la alteración en el funcionamiento fisario ${ }^{(39)}$.

Como limitaciones del presente estudio se destaca su naturaleza retrospectiva, el bajo número de pacientes y el corto seguimiento clínico radiológico para lograr evaluar las complicaciones a largo plazo (coxartrosis). Dada la edad más pequeña de algunos pacientes, no fue posible realizar el iHOT-12 completo. Sin embargo, el score se presenta como promedio de las 11 preguntas utili- 
zadas. Además, un único investigador realizó la medición clínica y radiográfica de los pacientes. Y como Loder ha transmitido en sus artículos sobre el tema, la falta de precisión en la definición de DEFP inestable podría sesgar los resultados ${ }^{(11)}$. Las fortalezas del estudio fueron el completo y real seguimiento clínico y radiológico de la serie analizada, con un mínimo de dos años, que nos permitió conocer las complicaciones más temidas de esta afección, siendo realizado en un centro de referencia ortopédico, donde se trata la amplia mayoría de los casos de DEFP del Uruguay. También fue posible medir la función de las caderas afectadas de forma objetiva por parte del examinador y de forma subjetiva mediante el score de calidad de vida, iHOT-12 y la remodelación femoral a través de las radiografías de control en todos los casos contactados.

\section{Conclusiones}

En este estudio se observa una elevada proporción de DEFP inestables. Los resultados clínicos de calidad de vida a través del PROM utilizado eran mejores en los pacientes que habían sufrido DEFP inestables en comparación con los DEFP estables. La ausencia de NAV en DEFP inestables y el elevado número de PFA en DEFP estables podría explicarnos estos hallazgos inesperados.

\section{Agradecimientos}

El equipo desea agradecer al personal que trabaja en el Archivo médico por ayudarnos con las historias clínicas y al personal de secretaría del servicio de ortopedia pediátrica del Centro Hospitalario Pereira Rossell, las Sras. Andrea Batalla y Esther Viera.

\section{Summary}

Introduction: the impact on the quality of life of patients with unstable and stable slipped capital femoral epiphysis is rather unknown. The study aims to learn about therapeutic outcomes in a quality of life score and the complications of the affected population in an orthopedics referecence center.

Method: this cohort study included 28 children treated at the Pereira Rossell Hospital Center between 2010 and 2016. Patients were assessed both clinically and with $\mathrm{x}$-rays, with a 2 -year minimum postoperative follow-up. The "International Hip Outcome Tool" (iHOT-12) questionnaire was used to measure the results reported by patients. Avascular necrosis, chondrolysis and femoroacetabular impingement were the complications evaluated by the study.

Results: the study identified 38 hips treated for slipped capital femoral epiphysis. 28 of them were classified as stable (74\%) and 10 as unstable (26\%). In-situ fi- xation was the most frequently used surgical treatment. Upon completion of follow-up, 27 patients were assessed and the iHOT12 showed a significant difference between stable and unstable slips (70 (range 38-95) and 86 (57-100); $\mathrm{P}=0,017)$ respectively. No avascular necrosis or chondrolysis were observed and femoroacetabular impingement was $19 \%$ ( $n=7$ hips; 6 stable and 1 unstable).

Conclusions: quality of life clinical results obtained by PROM were significantly better in the unstable slipping epiphysis of the proximal femur than in the stable epiphysis. Absence of avascular necrosis in unstable hips and the greater proportion of femoroacetabular impingements in stable slips despite a noticeable femoral remodeling following in-situ fixation could explain these surprising results.

\section{Resumo}

Introdução: o impacto sobre a qualidade de vida dos pacientes com deslizamentos de epífise proximal do fêmur instáveis e estáveis é pouco conhecido. O objetivo deste trabalho foi conhecer os resultados terapêuticos utilizando um indicador de qualidade de vida e as complicações da população afetada em um centro ortopédico de referência.

Materiais e métodos: este estudo de coorte incluiu 28 crianças tratadas no Centro Hospitalario Pereira Rossell no período 2010-2016. Foram avaliados os aspectos clínicos e radiológicos com um seguimento pós-operatório mínimo de 2 anos. O questionário International Hip Outcome Tool (iHOT-12) foi utilizado para medir os resultados descritos pelo paciente. Foram avaliadas as complicações por necrose avascular, condrólise e pinçamento femoroacetabular.

Resultados: foram identificados 38 quadris tratados por deslizamento epifisário proximal de fêmur. 28 foram classificados como estáveis (74\%) e 10 como instáveis $(26 \%)$. A fixação in situ foi o tratamento cirúrgico mais frequentemente utilizado. No término do seguimento 27 pacientes foram avaliados e o iHOT12 mostrou uma diferença significativa entre deslizamentos estáveis e instáveis (70 (intervalo 38-95) e 86 (57-100); $\mathrm{P}=0,017)$ respectivamente. Não foram observadas necrose avascular nem condrólise e o pinzamiento femoroacetabular foi de $19 \%$ ( $n=7$ quadris; 6 estáveis e 1 instável).

Conclusões: os resultados clínicos de qualidade de vida através do PROM (desfechos medidos pelo paciente) utilizado eram significativamente melhores nos DEFP instáveis comparados com os estáveis. A ausência de necrose avascular nos quadris instáveis e a maior proporção de pinçamento femoroacetabular nos deslizamentos estáveis apesar de uma remodelação femoral no- 
tória depois da fixação in situ, poderia explicar estes achados inesperados.

\section{Bibliografía}

1. Peck D. Slipped capital femoral epiphysis: diagnosis and management. Am Fam Physician 2010; 82(3):258-62.

2. Pritchett JW, Perdue KD. Mechanical factors in slipped capital femoral epiphysis. J Pediatr Orthop 1988; 8(4):385-8.

3. Hagglund G. Pinning the slipped and contralateral hips in the treatment of slipped capital femoral epiphysis. J Child Orthop 2017; 11(2):110-3.

4. Kroin E, Frank JM, Haughom B, Kogan M. Two cases of avascular necrosis after prophylactic pinning of the asymptomatic, contralateral femoral head for slipped capital femoral epiphysis: case report and review of the literature. J Pediatr Orthop 2015; 35(4):363-6.

5. Herngren B, Stenmarker M, Vavruch L, Hagglund G. Slipped capital femoral epiphysis: a population-based study. BMC Musculoskelet Disord 2017; 18(1):304.

6. Loder RT, O'Donnell PW, Didelot WP, Kayes KJ. Valgus slipped capital femoral epiphysis. J Pediatr Orthop 2006; 26(5):594-600.

7. Loder RT, Richards BS, Shapiro PS, Reznick LR, Aronson DD. Acute slipped capital femoral epiphysis: the importance of physeal stability. J Bone Joint Surg Am 1993; 75(8):1134-40.

8. Zaltz I, Baca G, Clohisy JC. Unstable SCFE: review of treatment modalities and prevalence of osteonecrosis. Clin Orthop Relat Res 2013; 471(7):2192-8

9. Ulici A, Carp M, Tevanov I, Nahoi CA, Sterian AG, Cosma D. Outcome of pinning in patients with slipped capital femoral epiphysis: risk factors associated with avascular necrosis, chondrolysis, and femoral impingement. J Internat Med Res 2018; 46(6):2120-7.

10. Lang P, Panchal H, Delfosse EM, Silva M. The outcome of in-situ fixation of unstable slipped capital femoral epiphysis. J Pediatr Orthop B 2019; 28(5):452-7.

11. Herngren B, Stenmarker M, Enskar K, Hagglund G. Outcomes after slipped capital femoral epiphysis: a population-based study with three-year follow-up. J Child Orthop 2018; 12(5):434-43.

12. Roaten J, Spence DD. Complications related to the treatment of slipped capital femoral epiphysis. Orthop Clin North Am 2016; 47(2):405-13.

13. Hosalkar HS, Pandya NK, Bomar JD, Wenger DR. Hip impingement in slipped capital femoral epiphysis: a changing perspective. J Child Orthop 2012; 6(3):161-72.

14. Ortegren J, Peterson P, Svensson J, Tiderius CJ. Persisting CAM deformity is associated with early cartilage degeneration after slipped capital femoral epiphysis: 11-year follow-up including dGEMRIC. Osteoarthritis Cartilage 2018; 26(4):557-63.

15. Larson AN, Sierra RJ, Yu EM, Trousdale RT, Stans AA. Outcomes of slipped capital femoral epiphysis treated with in situ pinning. J Pediatr Orthop 2012; 32(2):125-30.
16. de Poorter JJ, Beunder TJ, Gareb B, Oostenbroek HJ, Bessems GH, van der Lugt JC, et al. Long-term outcomes of slipped capital femoral epiphysis treated with in situ pinning. J Child Orthop 2016; 10(5):371-9.

17. Nectoux E, Décaudain J, Accadbled F, Hamel A, Bonin N, Gicquel P, et al. Evolution of slipped capital femoral epiphysis after in situ screw fixation at a mean 11 years' follow-up: a 222 case series. Orthop Traumatol Surg Res 2015; 101(1):51-4.

18. Escott BG, De La Rocha A, Jo CH, Sucato DJ, Karol LA. Patient-reported health outcomes after in situ percutaneous fixation for slipped capital femoral epiphysis: an average twenty-year follow-up study. J Bone Joint Surg Am 2015; 97(23):1929-34.

19. Sansone M, Ahlden M, Jónasson P, Thomeé C, Sward L, Ohlin A, et al. Outcome after hip arthroscopy for femoroacetabular impingement in 289 patients with minimum 2-year follow-up. Scand J Med Sci Sports 2017; 27(2):230-5.

20. Baumann F, Popp D, Muller K, Muller M, Schmitz P, Nerlich M, et al. Validation of a German version of the international hip outcome tool 12 (iHOT12) according to the COSMIN checklist. Health Qual Life Outcomes 2016; 14:3.

21. Thorborg K, Tijssen M, Habets B, Bartels EM, Roos EM, Kemp J, et al. Patient-Reported Outcome (PRO) questionnaires for young to middle-aged adults with hip and groin disability: a systematic review of the clinimetric evidence. $\mathrm{Br} \mathrm{J}$ Sports Med 2015; 49(12):812.

22. Cibere J, Thorne A, Bellamy N, Greidanus N, Chalmers A, Mahomed N, et al. Reliability of the hip examination in osteoarthritis: effect of standardization. Arthritis Rheum 2008; 59(3):373-81.

23. Klaue K, Durnin CW, Ganz R. The acetabular rim syndrome. A clinical presentation of dysplasia of the hip. J Bone Joint Surg Br 1991; 73(3):423-9.

24. Southwick WO. Osteotomy through the lesser trochanter for slipped capital femoral epiphysis. J Bone Joint Surg Am 1967;49(5):807-35.

25. Pring M, Adamczyk M, Hosalkar H, Bastrom T, Wallace D, Newton P. In situ screw fixation of slipped capital femoral epiphysis with a novel approach: a double-cohort controlled study. J Childr Orthop 2010; 4(3):239-44.

26. Notzli HP, Wyss TF, Stoecklin CH, Schmid MR, Treiber K, Hodler J. The contour of the femoral head-neck junction as a predictor for the risk of anterior impingement. J Bone Joint Surg Br 2002; 84(4):556-60.

27. Griffin DR, Dickenson EJ, Wall PDH, Achana F, Donovan JL, Griffin J, et al. Hip arthroscopy versus best conservative care for the treatment of femoroacetabular impingement syndrome (UK FASHIoN): a multicentre randomised controlled trial. Lancet 2018; 391(10136):2225-35.

28. Ficat RP. Idiopathic bone necrosis of the femoral head. Early diagnosis and treatment. J Bone Joint Surg $\mathrm{Br}$ 1985; 67(1):3-9.

29. Ruiz-Iban MA, Seijas R, Sallent A, Ares O, Marín-Peña O, Muriel A, et al. The international hip outcome tool-33 
(iHOT-33): multicenter validation and translation to Spanish. Health Qual Life Outcomes 2015; 13:62.

30. O'Brien ET, Fahey JJ. Remodeling of the femoral neck after in situ pinning for slipped capital femoral epiphysis. J Bone Joint Surg Am 1977; 59(1):62-8.

31. Dawes B, Jaremko JL, Balakumar J. Radiographic assessment of bone remodelling in slipped upper femoral epiphyses using Klein's line and the alpha angle of femoral-acetabular impingement: a retrospective review. J Pediatr Orthop 2011; 31(2):153-8.

32. Akiyama M, Nakashima Y, Kitano T, Nakamura T, Takamura K, Kohno Y, et al. Remodelling of femoral head-neck junction in slipped capital femoral epiphysis: a multicentre study. Int Orthop 2013; 37(12):2331-6.

33. Ortegren J, Bjorklund-Sand L, Engbom M, Tiderius CJ. Continued growth of the femoral neck leads to improved remodeling after in situ fixation of slipped capital femoral epiphysis. J Pediatr Orthop 2018; 38(3):170-5.

34. Murgier J, de Gauzy JS, Jabbour FC, Iniguez XB, Cavaignac $\mathbf{E}$, Pailhe $\mathbf{R}$, et al. Long-term evolution of slipped capital femoral epiphysis treated by in situ fixation: a 26 years follow-up of 11 hips. Orthop Rev (Pavia) 2014; 6(2):5335.

35. Loder RT. What is the cause of avascular necrosis in unstable slipped capital femoral epiphysis and what can be done to lower the rate? J Pediatr Orthop 2013; 33 (Suppl 1):S88-91.

36. Crepeau A, Birnbaum M, Vander Have K, Herrera-Soto J. Intracapsular pressures after stable slipped capital femoral epiphysis. J Pediatr Orthop 2015; 35(8):e90-2.

37. Hosseinzadeh P, Iwinski HJ, Salava J, Oeffinger D. Delay in the diagnosis of stable slipped capital femoral epiphysis. J Pediatr Orthop 2017; 37(1):e19-e22.

38. Murray AW, Wilson NI. Changing incidence of slipped capital femoral epiphysis: a relationship with obesity? J Bone Joint Surg Br 2008; 90(1):92-4.

39. Halverson SJ, Warhoover T, Mencio GA, Lovejoy SA, Martus JE, Schoenecker JG. Leptin elevation as a risk factor for slipped capital femoral epiphysis independent of obesity status. J Bone Joint Surg Am 2017; 99(10):865-72.

\section{Contribución de autores}

Juan Filippini, https://orcid.org/0000-0002-7912-265. Concepción, diseño, ejecución, análisis, interpretación de los resultados, redacción, revisión crítica.

Ignacio Pagano, https://orcid.org/0000-0003-4805-7543. Concepción, diseño, interpretación de los resultados, redacción, revisión crítica.

Johan von Heideken, https://orcid.org/0000-0003-3604-9167. Concepción, diseño, análisis, interpretación de los resultados, redacción, revisión crítica.

María Elena Pérez, https://orcid.org/0000-0001-5778-2267. Concepción, diseño, interpretación de los resultados, redacción, revisión crítica. 